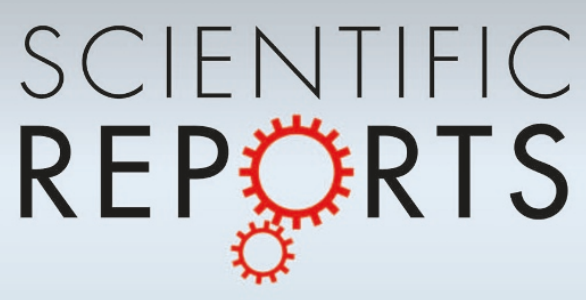

OPEN

SUBJECT AREAS:

SUPER-RESOLUTION

MICROSCOPY

PHASE-CONTRAST MICROSCOPY

CONFOCAL MICROSCOPY

OPTICAL IMAGING

Received

20 February 2013

Accepted

30 May 2013

Published

21 June 2013

Correspondence and requests for materials should be addressed to M.G. (marc.guillon@ parisdescartes.fr)

\section{STED microscope with Spiral Phase Contrast}

\author{
Marcel A. Lauterbach ', Marc Guillon ', Asma Soltani ${ }^{2} \&$ Valentina Emiliani'
}

'Wavefront Engineering Microscopy Group, Neurophysiology and New Microscopies Laboratory, CNRS UMR 8154, INSERM S603, University Paris Descartes, Sorbonne Paris Cité, Paris, France, ${ }^{2}$ Laboratory of Membrane Dynamics and Neurological Diseases, University Paris Descartes, CNRS UMR 8192, University Paris Descartes, Sorbonne Paris Cité, Paris, France.

Stimulated Emission Depletion (STED) microscopy enables superresolution imaging of fluorescently marked nano-structures in vivo. Biological investigations are often hindered by the difficulty of relating super-resolved structures to other non-labeled features. Here we demonstrate that the similarity in optical design of Spiral Phase Contrast (SPC) and STED microscopes allows straightforward implementation of a phase contrast channel into a STED microscope in widefield and scanning modes. This method allows dual imaging and overlay in two contrast modes in fixed and in living specimens, in which double labeling is especially challenging. Living GFP- and YPF-stained neurons are imaged in one label-free phase contrast and one high-resolution STED channel. Furthermore, we implement SPC in widefield and scanning modes demonstrating that scanning confocal SPC yields the highest optical contrast. The latter configuration can provide contour detection or highlights and shadows reminiscent of differential interference contrast.

\footnotetext{
S
} timulated Emission Depletion (STED) microscopy ${ }^{1}$ is a powerful technique for imaging biological specimens with high spatial and temporal resolution. STED microscopy can image dynamic processes in living cells $^{2}$, in tissue ${ }^{3}$, and even in living mice $e^{4}$. Spatial resolution up to $20 \mathrm{~nm}$ in biological samples ${ }^{5}$ and $2.4 \mathrm{~nm}$ in diamond ${ }^{6}$ was demonstrated, and temporal resolution of 200 frames per second was achieved ${ }^{7}$.

In STED microscopy, fluorescence ability of fluorophores is inhibited at the periphery of a diffraction-limited excitation spot. This is achieved by overlaying the excitation spot with a red-shifted toroidal "STED" spot, which inhibits fluorescence by stimulated emission. The torus ("donut") shape is typically obtained by a helical phase mask placed along the laser beam path or imaged onto the back focal plane of the objective $e^{8}$. The non-inhibited area shrinks to arbitrarily small dimensions with increasing intensity of the STED beam, thus enabling superresolution imaging.

Super-resolution fluorescence microscopy resolves specifically stained nano-structures; however, these structures do not always reveal the overall sample context, in particular when the structures of interest are sparse like vesicles, focal adhesions, or ion channels. Thus, monitoring morphology in a supplementary imaging channel may be required. For living samples, such monitoring could track morphological changes of cells and even pursue their viability.

In STED microscopy, adding a second fluorescence channel, although possible ${ }^{9,10}$, is not straightforward, even for confocal resolution, because the absorption spectrum of an additional marker must have minimal overlap with the wavelength of the intense STED beam to avoid background fluorescence and bleaching. This makes the choice of fluorophores and laser sources difficult, especially for live-cell imaging. Additional constraints are imposed by the high required alignment precision of dichroic beam splitters hindering their easy exchange between imaging modalities.

Alternative contrast techniques such as bright-field microscopy or Differential Interference Contrast (DIC) can be used. However, the former often fails to give sufficient contrast because biological samples are mainly transparent objects, and the latter requires additional optics (e.g. Wollaston prisms) in the optical path, which makes it unsuitable for combination with STED microscopy.

In this article, we demonstrate a solution for easily upgrading any STED microscope with Spiral Phase Contrast $^{11}$ (SPC). This offers an additional contrast channel, which - contrary to other phase contrast methods - integrates seamlessly into the STED microscope without reconfiguring the optical path to switch between contrast modes. Furthermore, SPC images can be perfectly overlaid with the fluorescence images.

In this technique, phase contrast is achieved by using a helical phase mask as a Fourier filter in the back focal plane of the objective (Fig. 1, see also Methods and Supplementary Material). SPC imaging with coherent illumination yields contour detection of phase objects ${ }^{11,12}$. It can also provide shadow effects with coherent ${ }^{13}$ 

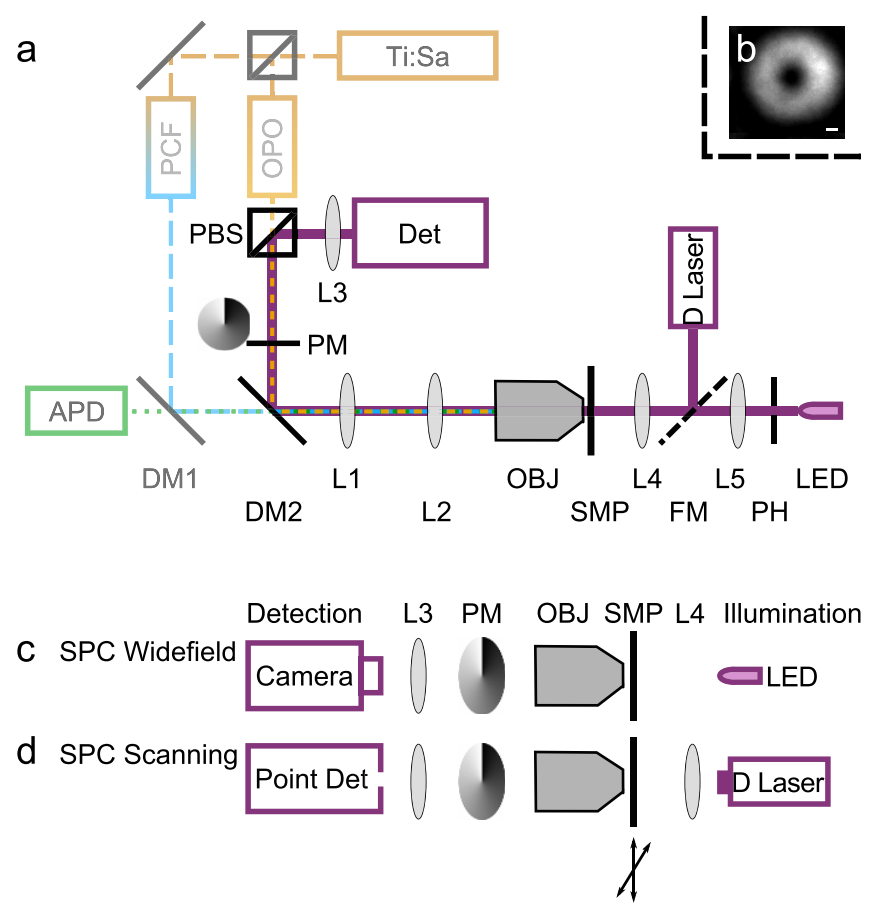

Figure 1 | Schematic diagram of the STED (Stimulated emission depletion) microscope with SPC (Spiral Phase Contrast). (a) For STED imaging, excitation $(480 \pm 10 \mathrm{~nm})$ and STED $(590 \pm 2 \mathrm{~nm}) /(620 \pm 2 \mathrm{~nm})$ beams are derived from one Ti:Sa laser, which pumps a photonic-crystal fiber (PCF) and an optical parametric amplifier (OPO). The STED beam passes a helical phase mask (PM), conjugated to the back pupil plane of the microscope objective OBJ. The sample is scanned with a piezo stage and fluorescence is detected by an avalanche photodiode APD. For SPC, an LED $(640 \pm 9 \mathrm{~nm})$ behind a pinhole $(\mathrm{PH})$, or a diode laser $(670 \mathrm{~nm})$, are chosen for illumination by a flip mirror (FM). Condenser lens L4 focuses the illumination into the sample. The phase-contrast detector (Det) can be a camera or a point detector. The polarizing beam splitter (PBS) separates the STED beam path and the phase contrast beam path. (b) When illuminating the sample with the diode laser, the image at the detector plane is a torus at the center of which the point detector is placed. Scale bar $1 \mu \mathrm{m}$.

(c)-(d): Schematic diagrams of SPC configurations: (c) Widefield detection (camera) with incoherent LED illumination. (d) Scanned confocal detection with focused laser illumination.

or incoherent illumination ${ }^{14}$ when the rotational symmetry of the optical system is broken. With this approach, chromosomes, erythrocytes and different types of cultured cells ${ }^{13}$ were visualized. Notably, the conventional design of a STED microscope allows
SPC imaging by the simple addition of a supplementary detector and an illumination source (Fig. 1 a). For generating the SPC image, light transmitted by the sample passes through the same helical phase mask used to shape the STED beam. We show that this configuration adds a phase contrast channel, taking advantage of the STED microscope's design without compromising high-resolution imaging capabilities. STED microscopy is combined here with two SPC modes: widefield and scanning (Fig. $1 \mathrm{c}$, d; see supplementary material for a detailed description of the different SPC configurations). Widefield SPC provides an overview of the sample on a camera with just an LED as illumination source. Scanning SPC enables perfect overlay with the fluorescence images and enhances background rejection when using confocal laser illumination. We demonstrate that this latter arrangement yields SPC with unprecedented contrast by imaging thin objects, such as neuronal growth cones and embedded astrocytes. Live-cell compatibility is shown by dual imaging with STED and SPC in living neurons expressing Lifeact-GFP ${ }^{15}$ and Channelrhodopsin-YFP ${ }^{16}$.

\section{Results}

Widefield SPC in a STED microscope. First, we demonstrate livecell SPC imaging in widefield mode (Fig. $1 \mathrm{c}$ ) in a STED microscope using an LED as light source. Because the LED is an extended incoherent source, alignment with the center of the phase mask is straightforward (Supplementary Fig. $1 \mathrm{c}$ ) although the phase-mask position is constrained by the optimization of the STED torus.

To highlight the sensitivity of SPC and its potential to enhance the utility of high-resolution live-cell imaging, we recorded flat growth cones, wherein the cytoskeleton was marked via transfection with Lifeact-GFP (Fig. 2). STED microscopy reveals the fine protrusions of a growth cone, which appears isolated (Fig. 2 a) because neighboring cells are not marked by transfection. Not relying on fluorescence, SPC reveals that the growth cone is not secluded but encounters an unstained neurite (Fig. 2 b). Actin filament bundles less than $80 \mathrm{~nm}$ are discerned with the GFP-based STED microscopy (Fig. $2 \mathrm{c}$ ), which is a common resolution for STED microscopy of fluorescent proteins ${ }^{17}$.

This experiment demonstrates simple and robust widefield SPC imaging in a STED microscope. However, as the source is spatially incoherent, complete destructive interference is never obtained at the detector plane, regardless of the presence or absence of a phase object in the sample plane. The phase contrast image has therefore a bright background, which intrinsically limits the contrast.

Confocal scanning SPC. To achieve enhanced contrast, we introduce confocal scanning SPC by using a focused coherent source (Fig. $1 \mathrm{~d}$ ). For this configuration, a diode-laser beam is focused to a spot in the sample plane by the condenser. The resulting intensity
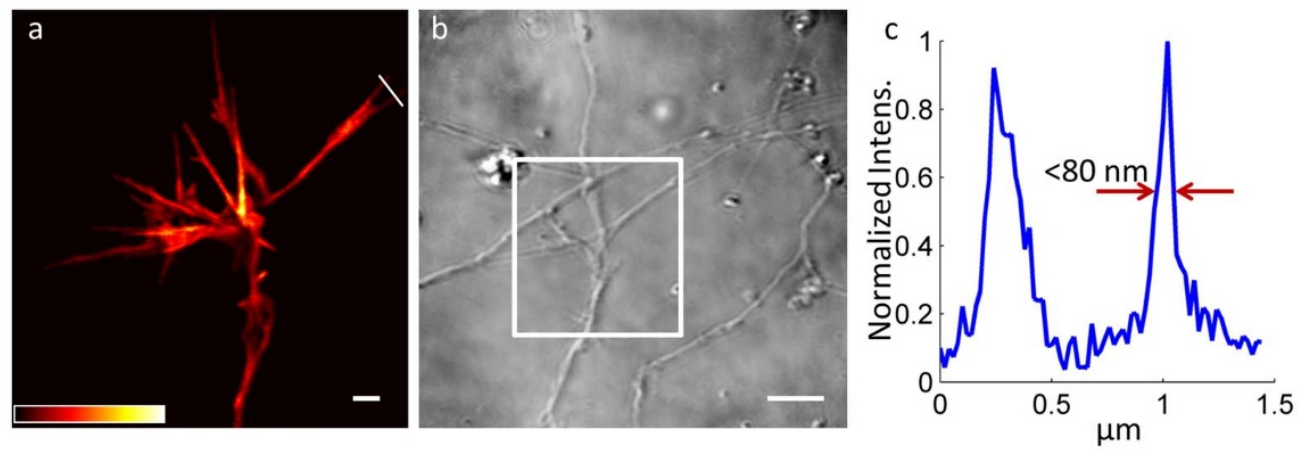

Figure $2 \mid$ Live-cell STED and SPC images obtained with incoherent LED illumination over a large area. STED imaging (a) visualizes fine details in a GFP-marked growth cone, while widefield SPC (b) shows that the growth cone interacts with surrounding unmarked neurites. The white box denotes the region imaged in (a). An intensity profile (c) along the white line in (a) confirms a resolution better than $80 \mathrm{~nm}$ in GFP stained neurons. Scale bars $1 \mu \mathrm{m}$ (a) and $5 \mu \mathrm{m}(\mathrm{b})$. The color scale is indicated in (a). 
profile at the detector plane is a torus (Fig. $1 \mathrm{~b}$ ) with a perfectly dark center with which the point detector is aligned. The phase contrast image is then recorded against a dark background, which reduces photon noise, leading to better signal-to-noise ratio and thus enabling detection of weaker phase objects. In addition, if the torus is exactly centered on the point detector isotropic phase contrast is obtained. Contours of objects are then highlighted independently of their orientation, which can be used for overlay with fluorescence images. Such isotropic contour detection against a dark background is shown in Fig. 3 a. A slight lateral displacement of the illumination spot shifts the torus relative to the point detector, thus breaking the rotational symmetry and producing highlights and shadows reminiscent of DIC (Fig. 3 b). Contours appear now with bas-relief effect. The background is no longer dark since the detector always receives some light from the torus rim. These data highlight that two different modes are available for confocal SPC. They also underline the high sensitivity of confocal SPC, which makes it suitable for imaging samples embedded in index-matched mounting medium. Astrocytes, which are particularly flat cells, are clearly delineated including fine protrusions. Figures $3 \mathrm{c}-\mathrm{e}$ show another flat structure, a fixed growth cone. Confocal fluorescence (Fig. 3 c) and more detailed STED (Fig. $3 \mathrm{~d}$ ) images reveal immunostained actin filaments, while SPC highlights the morphology of the growth cone. An overlay of the isotropic contour detection in SPC with the STED image shows how the actin cytoskeleton protrudes into the filopodia (Fig. 3 e).

Like widefield SPC, confocal SPC is also beneficial when imaging living samples. For instance an SPC image (Fig. 3 f) shows a network of living neurons in primary culture. This can serve also for selecting a region of interest for subsequent high-resolution imaging. This would be all the more beneficial if the neuronal network were not entirely recognizable with fluorescence microscopy due to sparse staining. Here, a dense staining was used for comparison. The fluorescence confocal image of the Channelrhodopsin-YFP stain in the same region (Fig. $3 \mathrm{~g}$ ) can be perfectly overlaid with the SPC image (Fig. $3 \mathrm{~h}$ ), since the same scanner was used for both recording modes. Live-cell STED microscopy of a region of interest resolves spine morphology, discerning necks smaller than $100 \mathrm{~nm}$ (Fig. 3 i), and revealing in addition the membranous distribution of Channelrhodopsin.

\section{Discussion}

We have presented straightforward implementation of Spiral Phase Contrast (SPC) into a STED microscope in two optical configurations based on widefield and scanning modes, respectively. This phase contrast technique uses the elements already present in the STED microscope and requires therefore almost no additional optics. Both SPC modes can serve as valuable second contrast channel for living and fixed samples in the high-resolution STED microscope. By introducing confocal SPC, we obtained high contrast even for flat cells embedded in index-matched mounting media. Additionally, SPC and STED imaging of GFP- and YFP-stained living neurons was achieved, verifying the live-cell compatibility of these techniques. For imaging fluorescent proteins with STED microscopy, a resolution below $80 \mathrm{~nm}$ was reached (Fig. 2 b). With organic dyes the resolution was even below $50 \mathrm{~nm}$ (Supplementary Fig. $3 \mathrm{~d}$ ).

On the one hand, widefield SPC using an LED light source is straightforward. On the other hand, scanning SPC allows perfect overlay with the super-resolved images, since the same scanner is used. Scanning SPC can also be obtained with LED illumination (Supplementary Fig. 1 d) as shown in Supplementary Figs 2 d, 3 b, but improved sensitivity to weak phase objects is obtained in confocal scanning mode with laser illumination. For widefield SPC (Supplementary Fig. 1 a), collimated laser illumination ${ }^{11}$ can also be used, but generates interference introducing inhomogeneous background $^{13}$ (Supplementary Figs 2 a). A comparison of widefield SPC with other phase contrast modes was shown by Maurer et $\mathrm{al}^{13}$.
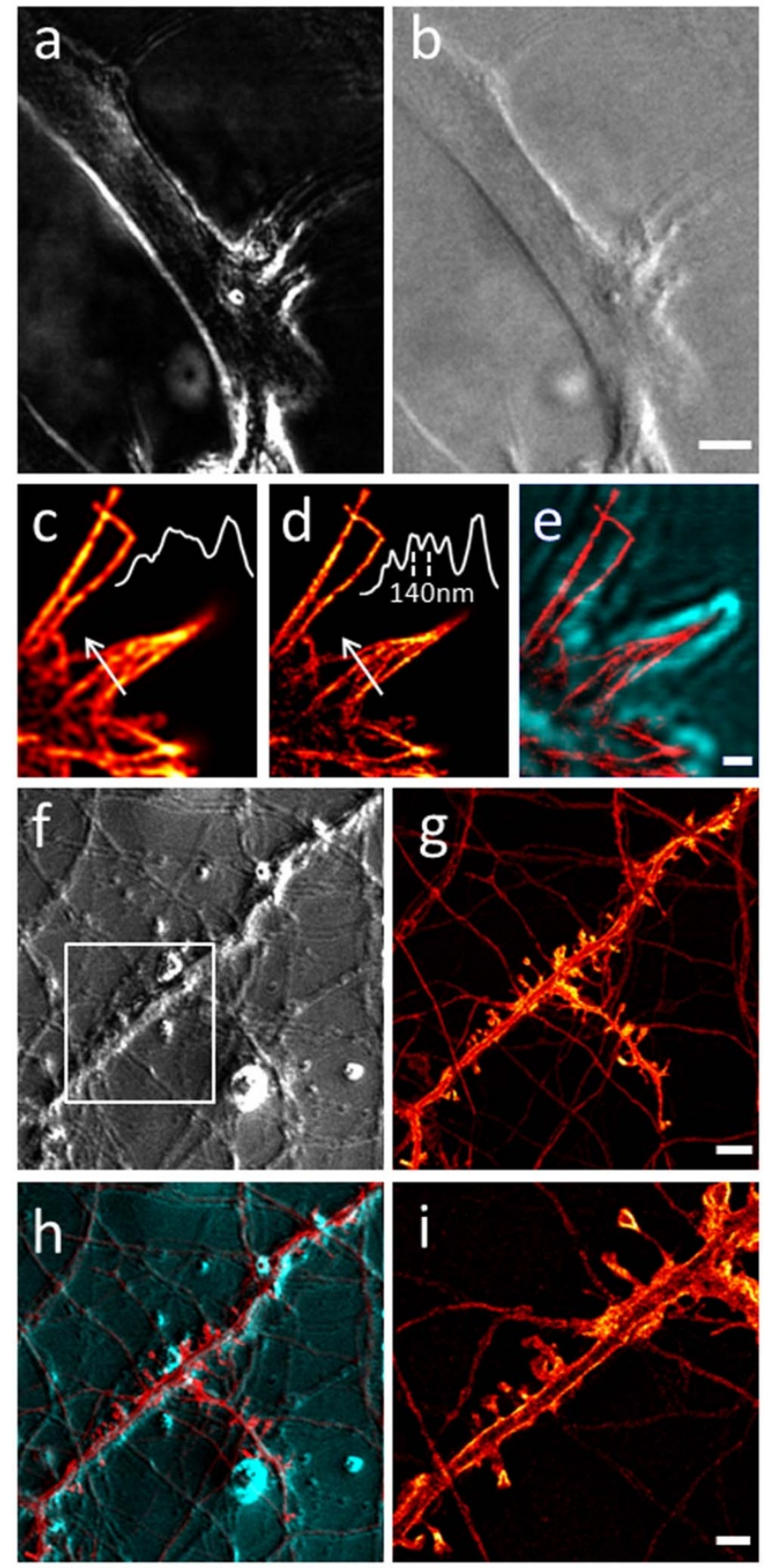

Figure 3 | Scanning confocal SPC visualizes weak phase objects.

(a) Symmetrical illumination highlights contours of an astrocyte in an indexmatched mounting medium on a dark background.

(b) Asymmetrical illumination shows the astrocyte with shadow effects on an elevated background as in DIC. (c) Immunostained actin filaments in the fine protrusions of neuronal growth cones (confocal fluorescence imaging). (d) STED imaging resolves details of the actin organization. Insets in (c) and (d) show intensity profiles along the white arrows. (e) Scanning confocal SPC (green) enables visualizing the fine protrusions in phase contrast. The symmetrical contour detection allows for an overlay with the STED image (red). (f) SPC visualizes the neuronal network of living neurons. (g) confocal fluorescence image. (h) SPC and confocal images are perfectly registered since they are recorded in the same scanner. (i) STED microscopy resolves spine morphology in YFP-transfected living neurons in the region indicated by the white box in (f). Scale bars: (b) $5 \mu \mathrm{m}$; (e) $0.5 \mu \mathrm{m}$; (g) $5 \mu \mathrm{m}$; (i) $2 \mu \mathrm{m}$. Panels (c), (d), (g), (i) use the same color scale as indicated in Fig. 2 a. 
Although STED microscopy resolves fine details in the sample (Figs 2 a, c, 3 d, i), including even YFP-marked spines in living neurons (Fig. 3 i), it relies strictly on fluorescent marking of the structure of interest. SPC visualizes in addition non-stained cells and thus puts highly resolved fluorescent structures into the specimen context (Fig. 2 b). Here, we showed that SPC can highlight surrounding neurites or cells that are not fluorescently marked. The visualization of non-stained objects can also be useful if the stained structure is too sparse to highlight the complete cell morphology. SPC can in this case reveal in which part of a cell and in which context a high-resolution fluorescence image was taken.

In the presented experiments, scanning SPC was performed by scanning the sample stage but should also be possible for beam scanning both with incoherent and coherent illumination. When illuminating the sample over a wide area with the LED, moving the sample by stage scanning is equivalent to moving the point detector relative to the sample by beam scanning. The whole field of view is always illuminated and no scanning of the illumination is necessary. In confocal configuration only a point in the sample is illuminated and therefore the illumination must be scanned synchronously with the detection. To this end, the illumination laser beam may be scanned through the objective. A retroreflector behind the sample can then refocus the illumination spot back to the same point as the incident beam ${ }^{18,19}$. Illumination and detection are thus performed through the objective and the light traverses the sample twice. In agreement with previous observation $\mathrm{s}^{20}$, we showed that confocal and widefield SPC remained possible even though the phase mask was wavelength mismatched by up to $80 \mathrm{~nm}$. In principle, it is therefore possible to record simultaneously on two detectors SPC and STED (or any other scanned, such as confocal fluorescence or RESOLFT $^{21}$ ) images.

\section{Methods}

Details of STED microscope with phase contrast. A home-built STED microscope was used that derives excitation and STED beams from a single laser pump (Fig. 1 a). This pump source (Ti:Sapphire Laser, Chameleon Ultra II, Coherent, Santa Clara, CA, USA) pumped a frequency doubled OPO (OPO PP Autom VIS-S1-830 fs, Coherent) to generate the STED beam (wavelength $620 \mathrm{~nm}$ for ATTO 532, $590 \mathrm{~nm}$ for GFP and YFP) and a photonic-crystal fiber PCF (femtoWHITE-800, NKT Photonics A/S, Birkerød, Denmark) to generate a supercontinuum for excitation. The femtosecond STED pulse was stretched by a pair of gratings to hundreds of picoseconds. Both beams were launched into mono-mode fibers (Thorlabs, New Jersey, USA; OZ Optics, Ontario, Canada) for spatial filtering; the fiber used for the STED beam was polarization maintaining. The STED beam passed a polarizing beam splitter for polarization cleaning and separation of the STED and the SPC beam paths. A dielectric filter (480/20, Chroma, Bellows Falls, VT, USA) was used for selecting the excitation wavelength (same filter for ATTO 532, GFP and YFP). Temporal overlay of the pulses was adjusted with an optical delay line. Excitation and STED beams were combined with dichroic mirror DM2 (Q570 DCSPXR, Chroma) and separated from fluorescence with DM1 (500 DCXRCT, Chroma). Fluorescence was detected by an avalanche photo diode (SPCM-AQRH13, Perkin Elmer, Fremont, CA, USA) behind a 550/50 filter (ATTO 532) or a 525/50 filter (GFP, YFP, both filters from Chroma). The sample was scanned with a piezo stage P734.2CL and digital controller E710 (Physik Instrumente, Karlsruhe, Germany). A single phase mask (VPP-A1, RPC Photonics, NY, USA) served for generating the toroidal STED focus and the phase contrast. It was conjugated to the back pupil plane of the objective (Olympus, Tokyo, Japan, $100 \times$, NA 1.40, UPLanSApo, Oil) by a telescope (L1, L2, focal lengths $125 \mathrm{~mm}$ ).

For SPC, we used as light sources either an LED (wavelength $639 \pm 9 \mathrm{~nm}$ ) or a diode laser (wavelength $670 \mathrm{~nm}$ ). Their wavelengths were chosen close to the STED wavelength so that all beams could pass through the same dichroics and match the design wavelength of the helical phase mask.

The diameter of the pinhole in front of the LED was $\approx 0.4 \mathrm{~mm}$. We estimated the coherence width of the illumination to be on the order of $\approx 3 \mu \mathrm{m}$. Collector lens L5 had a focal length of $100 \mathrm{~mm}$. The condenser lens L4 had focal length of $19 \mathrm{~mm}$. For confocal SPC, the diode laser was spatially filtered by a $20 \mu \mathrm{m}$ pinhole between $\mathrm{L} 4$ and L5 (not indicated in Fig. 1); the illuminated area in the sample was calculated to have a diameter of $2.5 \mu \mathrm{m}$. For phase contrast detection, a camera (FO124SB-FF, Foculus, New Electronic Technology GmbH, Finning, Germany) was used in widefield mode and a fiber coupled avalanche photo diode (SPCM-AQRH13, Perkin Elmer, Fremont, CA, USA) in scanning mode.
Sample preparation. Primary neuronal cultures were prepared from embryonic day (E) 16 mice embryos and cultured in glia-conditioned medium (adapted from Kaech et $\left.\mathrm{al}^{22}\right)$.

For live-cell imaging, cultured neurons were plated at 40000 cells per coverslip (\#1, BK-7, diameter 18 mm, Marienfeld Superior, Menzel-Gläser GmbH, Braunschweig, Germany) in glia-conditioned medium.

For staining of the actin cytoskeleton, neuronal transfections were performed with Lipofectamin 2000 (Invitrogen). For each well of a 12-well plate, a total of $2.5 \mu \mathrm{g}$ of Lifeact-GFP DNA was mixed with a $1: 2$ dilution of Lipofectamin 2000 in neurobasal medium and incubated at room temperature for $30 \mathrm{~min}$. Neurons at day in vitro (DIV) 11 were incubated for $3 \mathrm{~h}$ with the transfection mix and imaged the following day.

For Channelrhodopsin-YFP staining, neurons were transduced with AAV2/ 5.hSynapsin.hChR2(H134R).EYFP.WPREhGH $\left(2.065 \times 10^{10} \mathrm{GC} / \mathrm{mL}\right.$, UPenn vector core, Philadelphia, PA, USA) at DIV 12 and imaged 10 days after.

For fixed samples, cultured neurons were fixed at DIV 3 with pre-chilled methanol $\left(-20^{\circ} \mathrm{C}\right)$ and blocked with $0.2 \%$ FCS (fetal calf serum). For labeling the actin cytoskeleton, cells were incubated overnight with a primary antibody against beta-actin (Sigma-Aldrich, Lyon, France) at dilution 1:500 and subsequently with a secondary antibody against Mouse IgG (Merck Millipore, Molsheim, France) coupled to the organic dye ATTO 532 (ATTO-TEC, Siegen, Germany) at dilution $1: 200$ for $45 \mathrm{~min}$. Cells were embedded in phosphate buffered saline $(137 \mathrm{mM} \mathrm{NaCl}, 2.7 \mathrm{mM} \mathrm{KCl}$, $10 \mathrm{mM}$ phosphate, $\mathrm{pH} 7.4$ ), and samples were sealed with nail polish.

Cortical astrocytes were prepared from P0-1 NMRI mice as described previously ${ }^{23}$ The neocortex was dissected and mechanically dissociated. Cells were plated and maintained in Petri dishes for one week to reach confluence before their transfer to poly-L-ornithine treated cover slips (\#1, BK-7, Marienfeld Superior, Menzel-Gläser $\mathrm{GmbH}$ ). Cells were embedded in Vectashield HardSet Mounting Medium (Vector Laboratories, Inc., Burlingame, CA, USA). Cell media and supplements were purchased from Invitrogen (Cergy Pontoise, France).

Imaging parameters. Average excitation power for fluorescence images was 1.3 to $2.0 \mu \mathrm{W}$ in the aperture of the objective, STED power was 15 to $35 \mathrm{~mW}$. Pixel size for fluorescence images was $20 \mathrm{~nm}$, and dwell time $50 \mu$ s for small fields of view (Figs $2 \mathrm{a}$, $3 \mathrm{c}, \mathrm{d}, \mathrm{e}, \mathrm{i}$ ). Pixel size for the large fields of view (Figs $3 \mathrm{f}, \mathrm{g}, \mathrm{h}$ ) was $200 \mathrm{~nm}$ with $200 \mu \mathrm{s}$ dwell time. Confocal SPC images of astrocytes (Fig. 3 a, b) were recorded with $100 \mathrm{~nm}$ pixel size, and confocal SPC images of neurons with the same settings as the corresponding fluorescence images. Confocal SPC images of cells were smoothed by a $3 \times 3$ pixel median filter (small fields of view, Fig. 3 a, b, e) or a Gaussian filter (large fields of view, Fig. $3 \mathrm{~g}$, h) for display.

The widefield SPC images (Fig. 2 b, Supplementary Fig. 3 a) were flatfield corrected and filtered with a $3 \times 3$ pixel median filter.

The scanning LED-SPC image (Supplementary Fig. 3 b) was oversampled (50 nm pixel size) and smoothed by convolution with a Gaussian kernel of 3 pixels full width at half maximum.

The confocal SPC image for overlay in the region of interest (Fig. 3 e) was corrected for inhomogeneous background. Confocal and STED images were linearly deconvolved for display (Wiener filtering).

Intensity profiles (Figs 2 c, 3 c, d, Supplementary Fig. 3d) were determined on raw data, for the YFP stain with 4 pixels line width for averaging.

1. Hell, S. W. \& Wichmann, J. Breaking the diffraction resolution limit by stimulated emission: stimulated-emission-depletion fluorescence microscopy. Optics Letters 19, 780-782 (1994).

2. Westphal, V. et al. Video-rate far-field optical nanoscopy dissects synaptic vesicle movement. Science 320, 246-249 (2008).

3. Nägerl, U. V., Willig, K. I., Hein, B., Hell, S. W. \& Bonhoeffer, T. Live-cell imaging of dendritic spines by STED microscopy. Proceedings of the National Academy of Sciences of the United States of America 105, 18982-18987 (2008).

4. Berning, S., Willig, K. I., Steffens, H., Dibaj, P. \& Hell, S. W. Nanoscopy in a living mouse brain. Science 335, 551 (2012).

5. Donnert, G. et al. Macromolecular-scale resolution in biological fluorescence microscopy. Proceedings of the National Academy of Sciences of the United States of America 103, 11440-11445 (2006).

6. Wildanger, D. et al. Solid immersion facilitates fluorescence microscopy with nanometer resolution and sub-Ångström emitter localization. Advanced Materials 24, OP309-OP313 (2012)

7. Lauterbach, M. A., Ullal, C. K., Westphal, V. \& Hell, S. W. Dynamic imaging of colloidal-crystal nanostructurs at 200 frames per second. Langmuir 26, 1440014404 (2010).

8. Westphal, V., Lauterbach, M. A., Di Nicola, A. \& Hell, S. W. Dynamic far-field fluorescence nanoscopy. New Journal of Physics 9, 435 (2007).

9. Donnert, G. et al. Two-color far-field fluorescence nanoscopy. Biophysical Journal 92, L67-L69 (2007)

10. Tønnesen, J., Nadrigny, F., Willig, K. I., Wedlich-Söldner, R. \& Nägerl, U. V. Twocolor STED microscopy of living synapses using a single laser-beam pair. Biophysical Journal 101, 2545-2552 (2011).

11. Fürhapter, S., Jesacher, A., Bernet, S. \& Ritsch-Marte, M. Spiral phase contrast imaging in microscopy. Optics Express 13, 689-694 (2005). 
12. Davis, J. A., McNamara, D. E., Cottrell, D. M. \& Campos, J. Image processing with the radial hilbert transform: theory and experiments. Optics Letters 25, 99-101 (2000).

13. Maurer, C., Jesacher, A., Bernet, S. \& Ritsch-Marte, M. What spatial light modulators can do for optical microscopy. Laser and Photonics Reviews 5, 81-101 (2011).

14. Jesacher, A., Fürhapter, S., Bernet, S. \& Ritsch-Marte, M. Shadow effects in spiral phase contrast microscopy. Physical Review Letters 94, 233902 (2005).

15. Riedl, J. et al. Lifeact: a versatile marker to visualize F-actin. Nature Methods $\mathbf{5}$, 605-607 (2008).

16. Nagel, G. et al. Channelrhodopsin-2, a directly light-gated cation-selective membrane channel. Proceedings of the National Academy of Sciences of the United States of America 100, 13940-13945 (2003).

17. Willig, K. I., Rizzoli, S. O., Westphal, V., Jahn, R. \& Hell, S. W. STED microscopy reveals that synaptotagmin remains clustered after synaptic vesicle exocytosis. Nature 440, 935-939 (2006).

18. Art, J. J., Goodman, M. B. \& Schwartz, E. A. Simultaneous fluorescent and transmission laser scanning confocal microscopy. Biophysical Journal 59, 155a (1991).

19. Sheppard, C. J. R. \& Wilson, T. Multiple traversing of the object in the scanning microscope. Optica Acta 27, 611-624 (1980).

20. Situ, G., Pedrini, G. \& Osten, W. Spiral phase filtering and orientation-selective edge detection/enhancement. J. Opt. Soc. Am. A 26, 1788-1997 (2009).

21. Hell, S. W. Strategy for far-field optical imaging and writing without diffraction limit. Physics Letters A 326, 140-145 (2004).

22. Kaech, S. \& Banker, G. Culturing hippocampal neurons. Nature Protocols $\mathbf{1}$, 2406-2415 (2006)

23. Li, D., Hérault, K., Oheim, M. \& Ropert, N. FM dyes enter via a store-operated calcium channel and modify calcium signaling of cultured astrocytes. Proceedings of the National Academy of Sciences of the United States of America 106, 21960-21965 (2009).

\section{Acknowledgements}

We thank Karine Hérault for preparation of astrocyte cultures and Ouardane Jouannot for help with the Lifeact plasmid. We are grateful to Olivier Stettler, François Darchen, Dongdong Li, Nicole Ropert and Martin Oheim for helpful discussions. We thank Amanda Foust and Benoit Forget for careful reading of the manuscript and valuable suggestions. We acknowledge funding by the Paris School of Neuroscience (ENP). M.A.L. acknowledges support of the Center for Competences in nano-science (C'Nano) and that the research leading to these results has received funding from the People Programme (Marie Curie Actions) of the European Union's Seventh Framework Programme (FP7/2007-2013) under REA grant agreement no. PIEF-GA-2011-297917. We acknowledge Paris Descartes University for financial support (projet collaboratif).

\section{Author contributions}

M.L., M.G., V.E. designed research; M.L., M.G., A.S. performed experiments; M.L., M.G. analyzed data; M.L., M.G., V.E. wrote the paper; V.E. supervised the project.

\section{Additional information}

Supplementary information accompanies this paper at http://www.nature.com/ scientificreports

Competing financial interests: The authors declare no competing financial interests.

How to cite this article: Lauterbach, M.A., Guillon, M., Soltani, A. \& Emiliani, V. STED microscope with Spiral Phase Contrast. Sci. Rep. 3, 2050; DOI:10.1038/srep02050 (2013).

(c) (i) $(-)$ This work is licensed under a Creative Commons Attribution-

No NonCommercial-NoDerivs 3.0 Unported license. To view a copy of this license, visit http://creativecommons.org/licenses/by-nc-nd/3.0 